\title{
OPERATORS COMMUTING WITH THE SHIFT ON SEQUENCE SPACES
}

\author{
J. PRADA \\ Received 13 October 2003
}

\begin{abstract}
A complete characterization of shift-invariant operators that are isomorphisms is given in certain sequence spaces. Also given is a sufficient condition for an operator commuting with a shift-invariant operator to be shift invariant.
\end{abstract}

2000 Mathematics Subject Classification: 47B37, 46A45.

1. Introduction. There is a long-standing interest in linear continuous operators commuting with the right shift operator, weighted or not. The study of these operators is closely related to the study of operators commuting with the differentiation operator (weighted left shift). Several authors have treated topics connected with these operators; for instance, for unweighted shifts, a good reference is [10], while a good source for weighted shift operators is the papers [13, 14, 15] and the book of Halmos [9].

The concrete problem of determining the spectrum of a weighted right shift operator was studied mainly by Gellar $[3,4,5]$. There is a strong relationship between the spectrum of such an operator and the question of whether or not an operator which commutes with it is an isomorphism. In [17] the spectrum of the differentiation operator on certain sequence spaces was computed directly, although it could have been deduced using $[4$, theorem 10].

In the so-called umbral calculus appears the concept of a delta operator [2], which is invariant by differentiation and so connected with shift-invariant operators. In fact, in [2], the relationship between Sheffer operators, differentiation-invariant operators, and shift-invariant operators, as well as the importance of the spectrum in the characterization of isomorphisms, was shown. Similar questions were studied in the papers [6, 7, 8]; for differentiation-invariant operators, see [1, 2, 12, 16].

In the present paper, we consider shift-invariant operators on infinite power series spaces. Necessary and sufficient conditions for an operator to be continuous are given for any infinite power series space. Also given is a complete characterization of isomorphisms when the space is nuclear and a projective limit of Banach algebras. In addition, we give a sufficient condition for an operator commuting with a shift-invariant operator also to be shift invariant.

2. Definitions and notations. Let $\Lambda_{\infty}(\alpha)$ be the infinite power series space with the usual topology, that is, 


$$
\begin{gathered}
\Lambda_{\infty}(\alpha)=\left\{\left(x_{n}\right), x_{n} \in C, \sum_{n=0}^{\infty}\left|x_{n}\right| e^{k \alpha_{n}}<\infty, k=1,2,3, \ldots\right\}, \\
\left\|\left(x_{n}\right)\right\|_{k}=\sum_{n=0}^{\infty}\left|x_{n}\right| e^{k \alpha_{n}},
\end{gathered}
$$

where $\alpha_{0} \leq \alpha_{1} \leq \cdots \leq \alpha_{n} \leq \cdots \rightarrow \infty . \Lambda_{\infty}(\alpha)$ is a Fréchet space with a canonical basis, noted by $\left(e_{n}\right)$; its topological dual can be identified with the sequence space

$$
\Lambda_{\infty}^{\times}(\alpha)=\bigcup_{k=1}^{\infty} l^{\infty}\left(e^{-k \alpha_{n}}\right)
$$

and the coordinate operators are continuous. These spaces are nuclear if and only if

$$
\forall k, \quad \exists N(k) \text { such that }\left(\frac{e^{k \alpha_{n}}}{e^{N(k) \alpha_{n}}}\right) \in l^{1} .
$$

They are projective limits of the Banach spaces $l^{1}\left(e^{k \alpha_{n}}\right), k \in \mathbb{N}$, which are Banach algebras (with the convolution multiplication) if and only if there exists $C>0$ such that $\alpha_{n+m} \leq C+\alpha_{n}+\alpha_{m}$, for every $m$ and $n$. Well-known examples of nuclear infinite power series spaces that are projective limits of Banach algebras are the space $H(C)$ of entire functions on the complex plane (in this case, $\alpha_{n}=n$ ) and $s$, the space of sequences rapidly decreasing to zero (for $\alpha_{n}=\ln n$ ).

$S$ denotes the shift operator $S\left(e_{n}\right)=e_{n+1}$; it is assumed that $S$ is a continuous operator from $\Lambda_{\infty}(\alpha)$ to $\Lambda_{\infty}(\alpha)$, so we have the condition $\sup \alpha_{n+1} / \alpha_{n}<\infty$.

\section{Continuous operators commuting with $S$}

THEOREM 3.1. A linear operator $T$ is continuous on $\Lambda_{\infty}(\alpha)$ and commutes with $S$ if and only if

$$
T=\sum_{s=0}^{\infty} t_{s} S^{s}
$$

and satisfies the following condition: for all $k$, there exists $N(k)$ such that

$$
\sup _{n}\left(\sum_{s=0}^{\infty}\left|t_{s}\right| \frac{e^{k \alpha_{s}+n}}{e^{N(k) \alpha_{n}}}\right)<\infty
$$

Proof. Observe that the matrix $\left(t_{s, k}\right)_{s, k=0}^{\infty}$ defining $T$ is lower triangular, $t_{s, k}=0$, $k>s$, and $t_{s, k}=t_{s-k}, k \leq s$.

Therefore compute $T e_{n}$ for all $n$ and the result follows. 
Condition (3.2) can be simplified for certain infinite power series spaces as it is shown in the propositions below whose proofs are omitted.

Proposition 3.2. Assume that $\Lambda_{\infty}(\alpha)$ is a nuclear space. Then condition (3.2) is equivalent to the following one: for all $k$, there exists $N(k)$ and there exists $C(k)>0$ such that

$$
\left|t_{s}\right| \leq C(k) \frac{e^{N(k) \alpha_{n}}}{e^{k \alpha_{n+s}}}, \quad \forall s, n
$$

Proposition 3.3. Assume that $\Lambda_{\infty}(\alpha)$ is a nuclear space and a projective limit of Banach algebras. Then conditions (3.2) and (3.3) are equivalent to saying that the sequence $\left(t_{s}\right)$ is an element of $\Lambda_{\infty}(\alpha)$.

Observing that if $T$ commutes with $S, T\left(x_{s}\right)=\left(t_{s}\right) *\left(x_{s}\right)$, where $*$ represents the convolution product, (or $\left.T\left(\sum x_{s} z^{s}\right)=\sum t_{s} z^{s} \cdot \sum x_{s} z^{s}\right)$ [4, theorem 2], then we have the following proposition.

Proposition 3.4. If $T$ is a continuous linear operator on $\Lambda_{\infty}(\alpha)$ commuting with $S$, then the function $\phi(z)=\sum t_{s} z^{s}$ is holomorphic on a disk whose radius (finite or not) is greater than or equal to 1 .

Proof. If $T$ is continuous, condition (3.3) is true, and so

$$
\left|t_{s}\right| \leq C(k) \frac{e^{N(k) \alpha_{0}}}{e^{k \alpha_{s}}}, \quad \forall s .
$$

Therefore

$$
\limsup \left|t_{s}\right|^{1 / s} \leq e^{-k \liminf \alpha_{s} / s} \text {. }
$$

As $\liminf \alpha_{s} / s=0$, or is equal to a number $a>0$, or $\infty$, the result follows.

It is obvious that two operators commuting with $S$ commute with each other [4, Corollary 1], but it is not true, in general, that if $T_{1}$ commutes with $T_{2}$ and $T_{2}$ commutes with $S$, it follows that $T_{1}$ commutes with $S$; take $T_{2}=S^{2}$ and $T_{1}$ given by an infinite two-block matrix $\left(\begin{array}{cc}a_{00} & a_{01} \\ a_{10} & a_{11}\end{array}\right)$, where $a_{01}, a_{10}$ are different from zero and $a_{00} \neq a_{11}$. We show in the following proposition that for certain operators $T_{2}$ the result is true.

THEOREM 3.5. Let $T_{2}$ be a continuous linear operator from $\Lambda_{\infty}(\alpha)$ to $\Lambda_{\infty}(\alpha)$ commuting with $S, T_{2}=\sum_{s=0}^{\infty} t_{s} S^{s}$, verifying the condition that the sequence $t^{(0)}=\left(t_{1}, t_{2,} t_{3}, \ldots\right)$, $t^{(1)}=\left(0, t_{1}, t_{2}, t_{3}, \ldots\right), t^{(2)}=\left(0,0, t_{1}, t_{2}, t_{3}, \ldots\right), \ldots$ is a basis of the power series space $\Lambda_{\infty}(\alpha)$.

Then any linear continuous operator $T_{1}$ commuting with $T_{2}$ commutes with $S$.

Proof. The matrix corresponding to the operator $T_{2}$ verifies $t_{s, k}=0, k>s$, and $t_{s, k}=t_{s-k}, k \leq s$. Then, if $\left(a_{s, k}\right)_{s, k=0,1,2, \ldots}$ is the matrix of the operator $T_{1}$, we have the 
following equations (a consequence of $\left.\left(a_{s, k}\right) \times\left(t_{s, k}\right)=\left(t_{s, k}\right) \times\left(a_{s, k}\right)\right)$ :

$$
\begin{gathered}
\sum_{k=j+1}^{\infty} a_{0, k} t_{k-j, 0}=0, \quad j=0,1,2,3, \ldots, \\
t_{1,0} a_{0, j}+t_{0,0} a_{1, j}=\sum_{k=j}^{\infty} a_{1, k} t_{k-j, 0}, \quad j=0,1,2, \ldots, \\
t_{2,0} a_{0, j}+t_{1,0} a_{1, j}+t_{0,0} a_{2, j}=\sum_{k=j}^{\infty} a_{2, k} t_{k-j, 0}, \quad j=0,1,2, \ldots,
\end{gathered}
$$

and so on.

Observe that the operator given by the matrix $\left(a_{0,0}, a_{0,1}, a_{0,2}, \ldots\right)$ is continuous because of the continuity of $T_{1}$ and so an element of $\Lambda_{\infty}^{\times}(\alpha)$; therefore, as $\sup \alpha_{n+1} / \alpha_{n}<$ $\infty$, the element $\left(a_{0,1}, a_{0,2}, \ldots\right)$ belongs to $\Lambda_{\infty}^{\times}(\alpha)$ too. As $t^{(0)}, t^{(1)}, t^{(2)}, \ldots$ is a basis of $\Lambda_{\infty}(\alpha)$, the first set of equations implies that $a_{0,1}=a_{0,2}=a_{0,3}=\cdots=0$. Then the second set of equations can be written as

$$
\begin{gathered}
t_{1,0} a_{0, j}+t_{0,0} a_{1, j}=\sum_{k=j}^{\infty} a_{1, k} t_{k-j, 0}, \quad j=0, \\
t_{0,0} a_{1, j}=\sum_{k=j}^{\infty} a_{1, k} t_{k-j, 0}, \quad j=1,2, \ldots
\end{gathered}
$$

Observing as before that $\left(a_{11}-a_{00}, a_{12}, a_{13}, \ldots\right)$ is an element of $\Lambda_{\infty}^{\times}(\alpha)$ (the operator given by the matrix $\left(a_{10}, a_{11}, a_{12}, \ldots\right)$ is continuous by the continuity of $\left.T_{1}\right)$, it follows that $a_{11}=a_{00}, a_{12}=a_{13}=a_{14}=\cdots=0$.

Proceeding in an analogous way, the third set of equations can be written as

$$
\begin{gathered}
t_{2,0} a_{0, j}+t_{1,0} a_{1, j}+t_{0,0} a_{2, j}=\sum_{k=j}^{\infty} a_{2, k} t_{k-j, 0}, \quad j=0, \\
t_{1,0} a_{1, j}+t_{0,0} a_{2, j}=\sum_{k=j}^{\infty} a_{2, k} t_{k-j, 0}, \quad j=1, \\
t_{2,0} a_{0, j}+t_{1,0} a_{1, j}+t_{0,0} a_{2, j}=\sum_{k=j}^{\infty} a_{2, k} t_{k-j, 0}, \quad j=2,3, \ldots,
\end{gathered}
$$

and we get $a_{21}=a_{10}, a_{22}=a_{11}, a_{23}=a_{24}=\cdots=0$.

Thus the operator $T_{1}$ is given by a matrix that commutes with the matrix of $S$.

It is then of interest to determine a basis in $\Lambda_{\infty}(\alpha)$ of the previous form; noting that $t^{(1)}=S t^{(0)}, t^{(2)}=S t^{(1)}, t^{(3)}=S t^{(2)}, \ldots$, it is enough to find isomorphisms between infinite power series spaces that commute with $S$; in fact, given such an isomorphism $T$, it follows that $T e_{0}=t^{(0)}, T e_{1}=T S e_{0}=S T e_{0}=S t^{(0)}=t^{(1)}, T e_{2}=T S e_{1}=S T e_{1}=S t^{(1)}=$ $S^{2} t^{(0)}=t^{(2)}, \ldots$ and obviously we get a basis. 
4. Isomorphisms commuting with $S$. In this section, we consider isomorphisms between infinite power series spaces (nuclear and projective limits of Banach algebras). A complete characterization of such isomorphisms that commute with $S$ is given. In [11], the problem was dealt with for the space of holomorphic functions on a disc and, in [8], in the study of Sheffer operators, for certain spaces of entire functions.

THEOREM 4.1. Assume that $\Lambda_{\infty}(\alpha)$ is a nuclear space and a projective limit of Banach algebras; let $T$ be a linear operator commuting with $S$. Then $T$ is an isomorphism if and only if any of the following two equivalent conditions is satisfied:

(1) the sequence $\left(t_{s}\right)$ belongs to $\Lambda_{\infty}(\alpha)$ and $\left(t_{s}\right)=e^{\left(g_{s}\right)}$, where $\left(g_{s}\right)$ is an element of $\Lambda_{\infty}(\alpha)$, that is, $\left(t_{s}\right)$ is an exponential (or invertible) element of the Banach algebra $l^{1}\left(e^{k \alpha_{n}}\right)$ for all $k$,

(2) the sequence $\left(t_{s}\right)$ belongs to $\Lambda_{\infty}(\alpha)$ and $\sum_{s=0}^{\infty} t_{s} z^{s} \neq 0$ on the maximal ideal space of the Banach algebra $l^{1}\left(e^{k \alpha_{n}}\right)$ for all $k$.

Proof. Assume that the operator $T=\sum_{s=0}^{\infty} t_{s} S^{s}$ is an isomorphism. Then the operator $T^{-1}$ commutes with $S$, so $T^{-1}=\sum_{s=0}^{\infty} r_{S} S^{s}$; as $\left(t_{s}\right)$ and $\left(r_{S}\right)$ belong to $\Lambda_{\infty}(\alpha)$ and $\left(t_{s}\right) *\left(r_{s}\right)=1$, it follows that $\left(t_{s}\right)$ is an invertible (and exponential) element of all Banach algebras $l^{1}\left(e^{k \alpha_{n}}\right)$, for all $k$ (see [2, page 88]).

Conversely, assume that $\left(t_{s}\right)$ belongs to $\Lambda_{\infty}(\alpha)$ and $\left(t_{s}\right)=e^{\left(g_{s}\right)},\left(g_{s}\right) \in \Lambda_{\infty}(\alpha)$. Then the operator $T=\sum_{s=0}^{\infty} t_{s} S^{s}$ is continuous; as $\left(t_{s}\right)$ is an exponential (invertible) element of all algebras $l^{1}\left(e^{k \alpha_{n}}\right)$, taking $\left(r_{s}\right)=\left(t_{s}\right)^{-1}$, we have that $T^{-1}=\sum_{s=0}^{\infty} r_{s} S^{s}$ is continuous, implying that $T$ is an isomorphism.

Finally, note that conditions (1) and (2) are obviously equivalent as $\left\{x \in l^{1}\left(e^{k \alpha_{n}}\right)\right.$ : $\sum_{s=0}^{\infty} x_{s} z^{s} \neq 0$ for all $\left.|z| \leq e^{\rho}\right\}=\operatorname{inv} l^{1}\left(e^{k \alpha_{n}}\right)=\exp l^{1}\left(e^{k \alpha_{n}}\right)$, where $\rho=\lim _{n \rightarrow \infty} \log e^{k \alpha_{n}} / n$ (see [2, page 88]).

Observe that $\lim \alpha_{n} / n=0$ or is equal to a number $a>0$. Then the maximal ideal space of $l^{1}\left(e^{k \alpha_{n}}\right)$ is, in the first case, the closed disc $\bar{D}(1)$ (for all $k$ ) and, in the second case, $D\left(e^{k a}\right)$. Therefore condition (2) could be reformulated as follows:

(2) the sequence $\left(t_{s}\right)$ belongs to $\Lambda_{\infty}(\alpha)$ and $\sum_{s=0}^{\infty} t_{s} z^{s} \neq 0$ on $\bar{D}(1)\left(\right.$ if $\left.\lim \alpha_{n} / n=0\right)$ or on the whole complex plane (if $\lim \alpha_{n} / n>0$ ).

REMARK 4.2. In the conditions of the previous theorem, any exponential element of $\Lambda_{\infty}(\alpha)$ gives a basis; $e^{z}$ is a very simple example, and therefore the operator $I+$ $\sum_{s=1}^{\infty}(1 /(s-1) !) S^{s}$ satisfies the condition of Theorem 3.5.

REMARK 4.3. If $\Lambda_{\infty}(\alpha)=H(C)$, the isomorphisms are given by all entire functions without zeros on the complex plane; if $\Lambda_{\infty}(\alpha)=s$, all elements of $s$ without zeros on $\bar{D}(1)$ give isomorphisms (these include, in particular, all nonvanishing holomorphic functions on a disc with radius greater than 1).

\section{REFERENCES}

[1] J. Delsarte and J. L. Lions, Transmutations d'opérateurs différentiels dans le domaine complexe, Comment. Math. Helv. 32 (1957), 113-128 (French).

[2] A. Di Bucchianico, Probabilistic and Analytical Aspects of the Umbral Calculus, CWI Tract, vol. 119, Centrum voor Wiskunde en Informatica, Amsterdam, 1997. 
[3] R. Gellar, Cyclic vectors and parts of the spectrum of a weighted shift, Trans. Amer. Math. Soc. 146 (1969), 69-85.

[4] _ Operators commuting with a weighted shift, Proc. Amer. Math. Soc. 23 (1969), 538545.

[5]__ A generalization of weighted shifts, Indiana Univ. Math. J. 24 (1974), 259-264.

[6] S. Grabiner, Weighted shifts and Banach algebras of power series, Amer. J. Math. 97 (1975), 16-42.

[7] _ Dense subspaces of entire functions, Michigan Math. J. 33 (1986), no. 3, 417-422.

[8] Convergent expansions and bounded operators in the umbral calculus, Adv. in Math. 72 (1988), no. 1, 132-167.

[9] P. R. Halmos, A Hilbert Space Problem Book, D. Van Nostrand, New Jersey, 1967.

[10] H. Helson, Lectures on Invariant Subspaces, Academic Press, New York, 1964.

[11] I. I. Ibragimov and N. I. Nagnibida, The matrix method and quasi-power bases in the space of analytic functions in the disc, Russian Math. Surveys 30 (1975), no. 6, 107-154.

[12] N. I. Nagnibida, Isomorphisms of analytic spaces, commuting with the differentiation operator, Mat. Sb. (N.S.) 72 (114) (1967), 250-260 (Russian).

[13] N. K. Nikol'skiǔ, Invariant subspaces of weighted shift operators, Mat. Sb. (N.S.) 74 (116) (1967), 172-190 (Russian).

[14] _ Unicellularity and non-unicellularity of weighted shift operators, Dokl. Akad. Nauk SSSR 172 (1967), 287-290 (Russian).

[15] _ Basisness and unicellularity of weighted shift operators, Izv. Akad. Nauk SSSR Ser. Mat. 32 (1968), 1123-1137 (Russian).

[16] J. Prada, Operators commuting with differentiation, Math. Japon. 38 (1993), no. 3, 461-467.

[17] _ Delta operators on sequence spaces, Sci. Math. Jpn. 55 (2002), no. 2, 223-231.

J. Prada: Departamento de Matemáticas, Universidad de Salamanca, 37008 Salamanca, Spain E-mail address: prada@usa1.es 


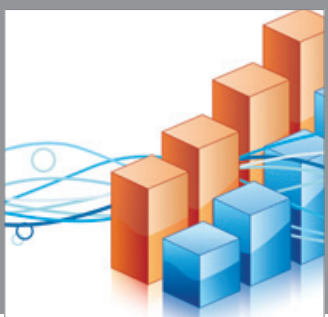

Advances in

Operations Research

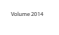

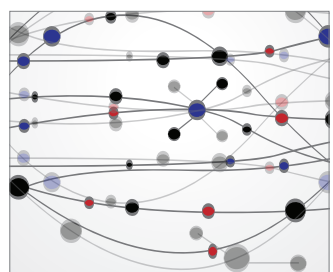

\section{The Scientific} World Journal
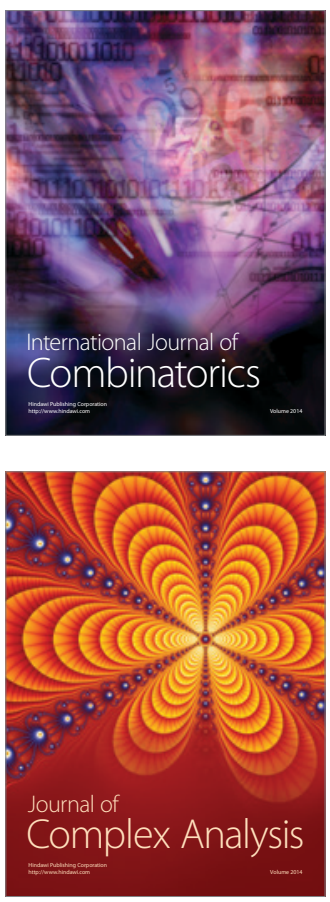

International Journal of

Mathematics and

Mathematical

Sciences
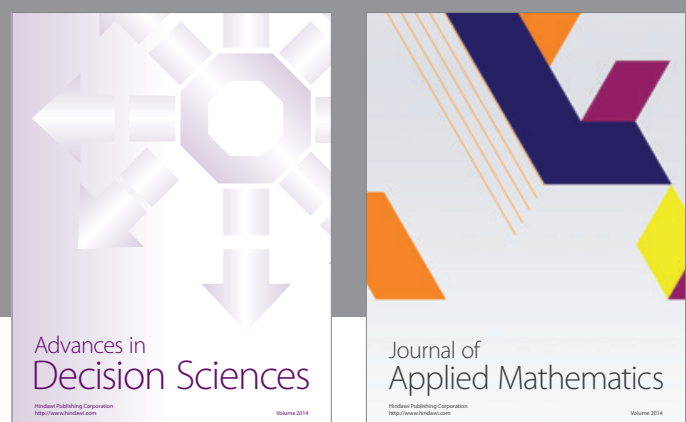

Journal of

Applied Mathematics
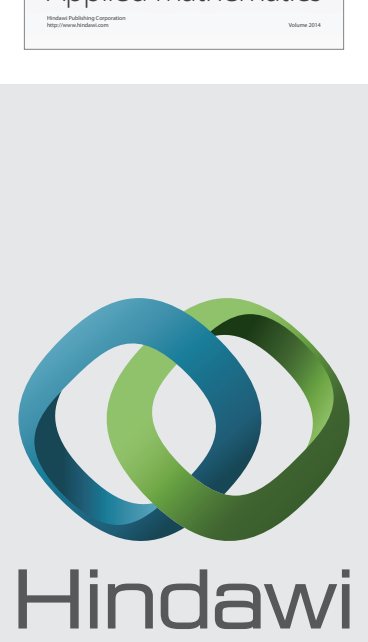

Submit your manuscripts at http://www.hindawi.com
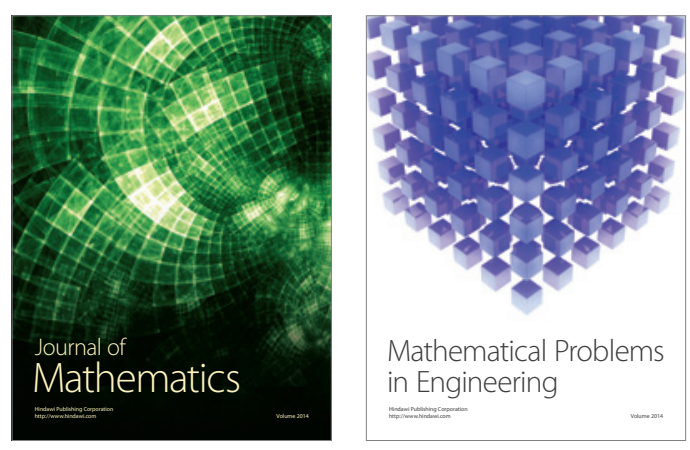

Mathematical Problems in Engineering
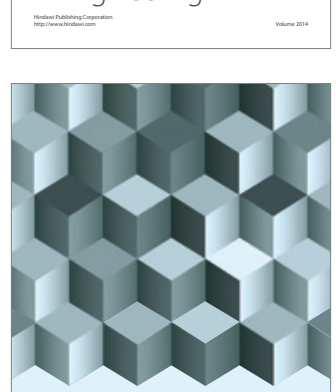

Journal of

Function Spaces
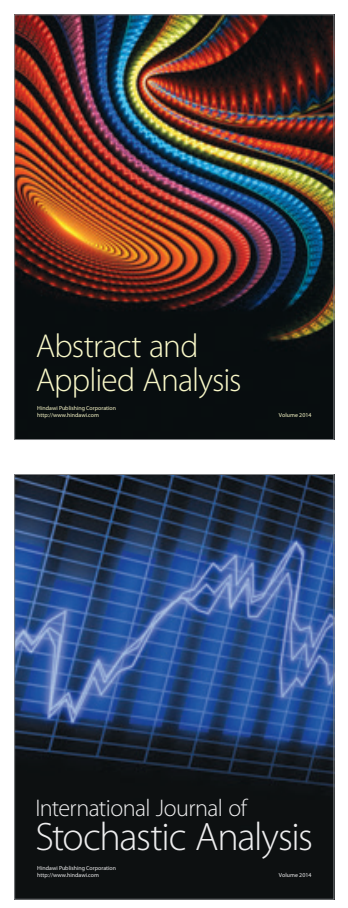

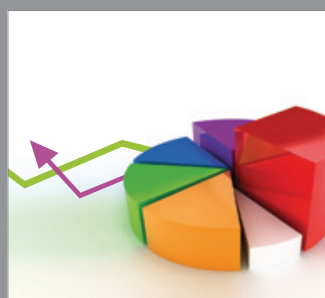

ournal of

Probability and Statistics

Promensencen
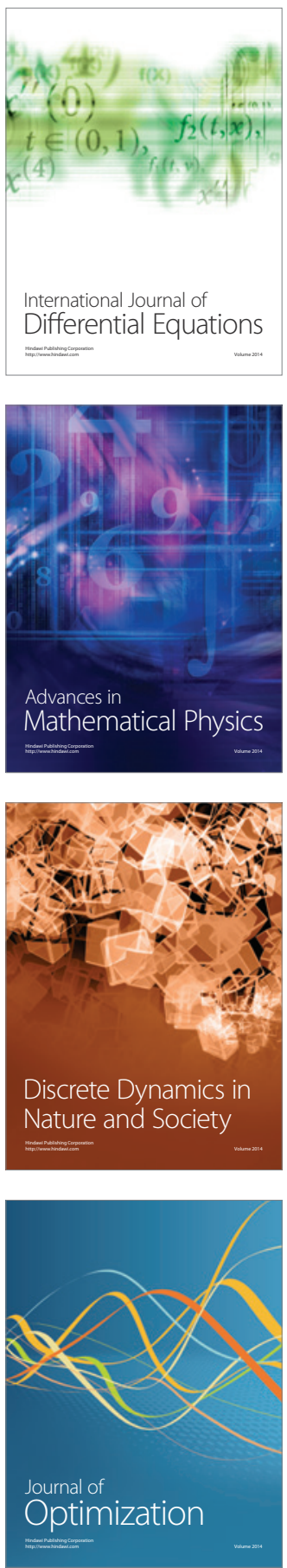